\title{
The Langer Lab's secret sauce
}

\section{Aimee L Hamilton}

$A$ s a researcher interested in how orgaAnizations thrive, I have been studying the Langer Lab as an exemplar for nearly six years. I have learned that the keys to success depend on recognizing that entrepreneurial science requires extensive collaborations on both the scientific side and the business side. Scientists that succeed are good collaborators; if you are a junior researcher, this primarily entails working hard, following up, being willing to take initiative and looking for opportunities to be a project champion.

\author{
Aimee L. Hamilton is assistant professor at \\ Daniels College of Business, University of \\ Denver, Denver, Colorado, USA. \\ e-mail:aimee.hamilton@du.edu
}

If you are a lab director, this means allowing others to share the credit and take direct ownership of the project, which is the necessary flip side to identifying a project champion. A project champion cannot do the job if someone above her or him is micromanaging the process or taking all the credit.

The Langer Lab is very flexible, especially with resources. This can mean working with what you have on hand rather than getting stymied by lack of resources. This is especially true with human talent. Back when the lab was just beginning, Langer conducted research primarily with the assistance of Massachusetts Institute of Technology undergraduates. The work needed to be done, and he used whatever talent he had (today, dozens of undergraduates still work alongside graduate students and postdocs in the lab).

The lab keeps an open mind about what might be a potential platform technology. Good ideas can come from anywhere and frequently arise through combined knowledge from two previously disparate lines of research. Just as it was uncharacteristic for a surgery lab in the 1970s to hire Langer, today his lab includes researchers not typically found in chemical engineering departments. He hires promising scientists even if the relevance of their expertise to current projects is not immediately apparent. This creates conditions in which ideas are shared and helps lead to multidisciplinary innovations.

\section{COMPETING FINANCIAL INTERESTS}

The author declares no competing financial interests.

\section{Startups on the menu}

In 2009, Kim Midwood, then at Imperial College London, participated in the London SciCafé following publication of her Nature Medicine article showing the role of tenascin C-mediated activation of toll-like receptor 4 in the maintenance of inflammation in models of arthritic joint disease (K. Midwood et al., Nat. Med. 15, 774-780 (2009)). At the meeting, she met several investors, and many subsequent discussions contributed to the creation of Nascient. Nature Biotechnology talked to her about her involvement in the company.

\section{SciCafé}

\section{NBT: What is the vision for Nascient?}

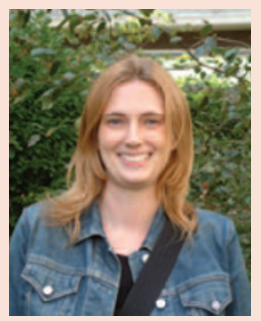

KM: The identification and development of antitenascin $\mathrm{C}$ antibodies that have real potential to provide meaningful benefit to patients with rheumatoid arthritis who are inadequately treated by existing commercial therapies. Nascient's main platform builds on 14 years of research investigating the biology of tenascin $\mathrm{C}$ and exploiting the novelty and strength of the intellectual property surrounding our work. Our primary aim presently is to further develop the underlying biology and facilitate its ready translation to antibody drug leads.
Nature Biotechnology: When did you decide it was time to start a company?

Kim Midwood: After publishing our paper, I was initially contacted by several large pharmaceutical companies interested in developing reagents to block the proinflammatory action of tenascin $\mathrm{C}$ as a potential therapeutic agent for the treatment of inflammatory diseases. Nascient was ultimately founded in the summer of 2012 as the first venture brought to fruition by SweetSpot Therapeutics, an Imperial Innovations-participated company that exploits the strengths of academic research by combining it with proven industrial capabilities in novel antibody-based therapeutics.
NBT: Have your entrepreneurial activities modified your approach to research?

KM: The Kennedy Institute of Rheumatology at Oxford has achieved great success in the development of new drugs for treating rheumatoid arthritis and continues to provide an environment that is incredibly supportive of translational research with cutting-edge basic science facilities as well as access to patient samples and our own clinical trials unit. Within Nascient, I act as founder [and] consultant, and in this way I can provide insight into how the science is driven forward to best achieve our translational goals. In return, my academic group carries out work in the lab to ensure we are tackling what most directly addresses clinical needs. 